\title{
Protective effects of Danggui Buxue Tang on renal function, renal glomerular mesangium and heparanase expression in rats with streptozotocin-induced diabetes mellitus
}

\author{
TAI-SHENG YE $^{1 *}$, YING-WEN ZHANG ${ }^{1 *}$ and XIAN-MEI ZHANG ${ }^{2}$ \\ ${ }^{1}$ Department of Integrated Traditional Chinese and Western Medicine, Zhongnan Hospital, Wuhan University, \\ Wuhan, Hubei 430071; ${ }^{2}$ Department of Integrated Traditional Chinese and Western Medicine, \\ The Third Hospital of Wuhan, Wuhan, Hubei 430060, P.R. China
}

Received January 26, 2015; Accepted March 8, 2016

DOI: $10.3892 /$ etm.2016.3218

\begin{abstract}
Danggui Buxue Tang (DBT) is a simple combination of Radix Astragali and Radix Angelica sinensis (5:1), with a variety pharmacological activities. In the present study, a single intravenous injection of $30 \mathrm{mg} / \mathrm{kg}$ streptozotocin and subsequent six weeks of high glucose diet in Sprague Dawley rats were used to induce diabetic nephropathy. Rats with diabetes mellitus showed increased levels of fasting blood glucose (FBG), blood urea nitrogen (BUN), serum creatinine (Scr), serum and urine $\beta 2$-microglobulins $(\beta 2-\mathrm{MG})$, and type IV collagen (all $\mathrm{P}<0.05$ ). DBT treatment significantly decreased the levels of FBG, BUN, Scr, serum and urine 32-MG, and type IV collagen. Furthermore, DBT treatment significantly and dose-dependently restored the ultrastructural injury, and reduced the expression of heparanase, compared with the vehicle $(\mathrm{P}<0.05)$. Therefore, DBT may be a novel therapeutic approach for the prevention and treatment of diabetic nephrology.
\end{abstract}

\section{Introduction}

Diabetic nephropathy is a serious and progressive complication of diabetes and occurs in 30-40\% of diabetic patients (1). Hyperglycemia is a crucial factor for the induction of diabetic nephropathy. Although positive effects on the development and progression of diabetic nephropathy may be produced through strict control of blood glucose, blood pressure and, in particular, blockade of the renin-angiotensin system (2), these

Correspondence to: Dr Ying-Wen Zhang, Department of Integrated Traditional Chinese and Western Medicine, Zhongnan Hospital, Wuhan University, 169 Donghu Road, Wuhan, Hubei 430071, P.R. China

E-mail: haohao3838@sina.com

*Contributed equally

Key words: Danggui Buxue Tang, renal function, glomerular ultrastructure, heparanase, diabetic nephropathy ainterventions are not sufficient to significantly reduce the high incidence of end-stage kidney damage caused by diabetes. Therefore, it is important to develop novel therapies that allow for the prevention and retardation of diabetic nephropathy.

The dried root (Radix) of Angelica sinensis is commonly known as Danggui, and is a crude drug widely used in traditional Chinese medicine for $>2,000$ years (3). Danggui is used to promote blood circulation for the treatment of menstrual disorders, to modulate the immune system, and the extracts of Radix A. sinensis have been found to exhibit various levels of antioxidant capacity (4). Radix Astragali, also known as Huangqi, is the dried root of Astragalus membranaceus, which belongs to the family of Fabaceae (5). Radix Astragali, which has also been used for the treatment of diabetes for hundreds of years in China, has been demonstrated to have an inhibitory effect on metal-induced oxidative stress (6). Danggui Buxue Tang (DBT) is a herbal decoction of Radix Astragali and Radix A. sinensis (5:1) (7). DBT is speculated to possess a variety of pharmacological actions, including regulation of immune functions, stimulation of red blood cell production and enhancement of cardiovascular function. Previous studies revealed that intake of DBT ameliorated the symptoms of diabetes and decreased the levels of blood glucose, plasma lipid, microalbuminuria, reduced the body weight and improved renal function in streptozotocin (STZ)-induced diabetic rats, indicating that DBT may be used as an adjuvant therapy in the prevention of diabetic nephropathy $(8,9)$.

The glomerular tuft, a network of tangled capillaries, is composed of three cell types: Endothelial cells at the inside of the capillary, podocytes on the outside of the capillary and glomerular mesangial cells (GMCs) supporting the capillary loops (10). GMCs occupy a central position in the renal glomerulus and are known to secrete extracellular matrix (ECM) proteins, such as type IV collagen, laminin and fibronectin (11). GMC proliferation, hypertrophy and progressive accumulation of ECM proteins lead to renal fibrosis, which is recognized to play a major role in progressive renal failure in diabetic nephropathy (12). Our previous study has provided in vitro evidence that DBT effectively inhibits high glucose-induced GMC proliferation and the expression of laminin, type IV collagen and fibronectin in GMCs (13). Whether DBT displays 
a protective effect on the ultrastructure of the renal glomerulus and particularly GMCs requires further investigation.

Heparanase (HPA) is an endoglucuronidase which functions at the cell-surface and within the ECM to degrade heparan sulfate, the main polysaccharide of the glomerular basement membrane (GBM) (14). Heparan sulfate is believed to play a major role in the charge-selective properties of the glomerular capillary wall, by binding to and assembling structural basement membrane proteins, and thus contributing to structural integrity and barrier function of the basement membrane (15). A recent study indicated that degradation and loss of heparan sulfate in the GBM and overexpression of HPA were closely linked to the development of diabetic nephropathy (16). However, whether DBT has beneficial effects on reversing the increased level of HPA in diabetes mellitus has not yet been elucidated.

Therefore, the aim of the present study was to investigate the effects of DBT on high glucose-induced ultrastructural alterations of the renal glomeruli in vivo and the expression of HPA in the renal cortex in a STZ-induced diabetic nephropathy rat model.

\section{Materials and methods}

Preparation of DBT. In preparation of DBT, exact quantities of Radix Astragali and Radix A. sinensis were weighed at a ratio of $5: 1$, and then mixed using a vortex. The herbs originated from Gansu province (China) and were obtained from the Department of Pharmacy of Zhongnan Hospital, Wuhan University (Wuhan, China). As previously described (17), the mixed herbs were extracted twice; initially boiled in water (1:6; $\mathrm{v} / \mathrm{w})$ for $1 \mathrm{~h}$, then the residue from first extraction was boiled in water (1:8) for $1.5 \mathrm{~h}$. Finally, the solutions were filtered using a rotary evaporator (RE-52A; Shanghai Ya Rong Biochemical Instrument Factory, Shanghai, China) and concentrated into doses of $0.5,1.0$ and $2.0 \mathrm{~kg} / \mathrm{l}$. The DBT decoction was stored at $4^{\circ} \mathrm{C}$ until required for experimentation.

Animals. Male Sprague Dawley (SD) rats $(n=60$; age, 4-6 weeks; weight, 170-200 g) were purchased from the Laboratory Animal Center of Wuhan University (Certificate No. SCXK 2003-00004). The rats were housed in a constant environment (temperature, $20-22^{\circ} \mathrm{C}$; humidity, 40-60\%) under a 12-h light/dark cycle. This study was approved by the Committee on the Use of Live Animal in Teaching and Research of the Wuhan University, and all the animal experiments were conducted in accordance with the stipulations of the Guide for the Care and Use of Laboratory Animals (18).

Experimental protocols and grouping. First, 52 SD rats were fed a high-fat and high-glucose diet (Wuhan Biological Engineering Co., Ltd., Wuhan, China) for two weeks, followed by fasting for $12 \mathrm{~h}$ (17). Then, the rats were administered a single intravenous injection of STZ (30 mg/kg; Sigma-Aldrich, St. Louis, MO, USA) and continuously received the high-fat and high-glucose diet. The rats were fasted for $12 \mathrm{~h}$, and the diabetic status was confirmed by monitoring blood glucose $72 \mathrm{~h}$ after the injection using a Johnson blood glucose meter (Johnson \& Johnson Medical Equipment Co., Ltd., Shanghai, China). Rats with fasting tail-blood glucose concentrations $\geq 7.0 \mathrm{mmol} / 1$ were considered to have developed diabetes mellitus (19). The rats that were confirmed with STZ-induced diabetes mellitus were randomly divided into five groups, treated once per day with the follows: $2 \mathrm{ml}$ normal saline (Vehicle group); low-dose (0.5 kg/l) DBT (DBT-L group); medium-dose $(1.0 \mathrm{~kg} / \mathrm{l})$ DBT (DBT-M group); high-dose (2.0 kg/l) DBT (DBT-H group); or $1.0 \mathrm{mg} / \mathrm{ml}$ gliclazide (Gliclazide group; Beijing Hailian Pharmaceutical Co., Ltd., Beijing, China), respectively. Gliclazide is an oral anti-diabetic drug and classified as a sulfonylurea, and thus has been used as a positive control previously (20). It was expected that $\sim 9$ rats were to be assigned into each group, based on our previous study (9). The dose selection was based on the conversion to the human equivalent dose (21). In addition, the remaining 8 rats that were fed with a normal diet and injected with normal saline instead of STZ, thus serving as a normal control group (NC group). Intragastric administration of normal saline, DBT or gliclazide was performed on the designated groups of rats once daily for 6 weeks. All rats were subsequently fasted overnight and sacrificed via an overdose intravenously injected pentobarbital $(100 \mathrm{mg} / \mathrm{kg})$ after the samples were collected. The kidneys were removed for the evaluation of ultrastructural changes by electron microscopy and immunohistochemistry for HPA expression.

Serum and urine studies. A 24-h urine collection was performed using metabolic cages (Wuhan Biological Engineering Co., Ltd.). Fasting blood glucose (FBG), blood urea nitrogen (BUN), serum creatinine (Scr) and serum and urine $\beta 2$-microglobulins ( $\beta 2-\mathrm{MG}$ ) levels were measured using an automatic biochemical analyzer (Hitachi 7020; Hitachi, Ltd., Tokyo, Japan). Type IV collagen detection kits were purchased from Wuhan Boster Biological Technology, Ltd. (Wuhan, China).

Electron microscopy for ultrastructure of the renal glomerular mesangium. For electron microscopy, the renal cortex was cut into $5 \times 5 \times 5 \mathrm{~mm}$ pieces and fixed in $2.5 \%$ glutaraldehyde (Wuhan Biological Engineering Co., Ltd.) for $2 \mathrm{~h}$. After washing three times in $0.1 \%$ phosphate-buffered saline (Wuhan Biological Engineering Co., Ltd.), all kidney specimens were fixed in $1 \%$ osmium tetroxide phosphate buffer solution (pH 7.2-7.4; Wuhan Biological Engineering Co., Ltd.) for $1.5 \mathrm{~h}$. Dehydration was performed by sequential exposure to 50,70 and $80 \%$ ethanol for $20 \mathrm{~min}$ each, and $100 \%$ ethanol twice for $15 \mathrm{~min}$ each. Sections were then embedded in epoxy resin (Wuhan Biological Engineering Co., Ltd.) and cut into ultrathin sections, which were stained with uranyl acetate and lead citrate (Wuhan Biological Engineering Co., Ltd.), and examined using an H-600 transmission electron microscope (Hitachi, Ltd.).

Immunohistochemistry for HPA expression. For immunohistochemistry for HPA expression, the renal cortex was cut into a $0.2-\mathrm{cm}$ block, which was fixed in $10 \%$ formalin (Wuhan Biological Engineering Co., Ltd.) and embedded in paraffin (Wuhan Biological Engineering Co., Ltd.). Then, the embedded blocks were cut into $4-\mu \mathrm{m}$ sections, which were dehydrated in graded ethanol and incubated with a primary rabbit anti-rat HPA monoclonal antibody (1:100; BA1630; 
Table I. Effects of DBT on fasting blood glucose, serum creatinine and blood urea nitrogen in rats.

\begin{tabular}{|c|c|c|c|c|c|c|c|}
\hline \multirow[b]{2}{*}{ Group } & \multirow[b]{2}{*}{$\mathrm{n}$} & \multicolumn{2}{|c|}{$\begin{array}{c}\text { Fasting blood } \\
\text { glucose (mmol/l) }\end{array}$} & \multicolumn{2}{|c|}{$\begin{array}{c}\text { Serum } \\
\text { creatinine }(\mu \mathrm{mol} / \mathrm{l})\end{array}$} & \multicolumn{2}{|c|}{$\begin{array}{c}\text { Blood urea } \\
\text { nitrogen (mmol/l) }\end{array}$} \\
\hline & & $\begin{array}{l}\text { Before } \\
\text { treatment }\end{array}$ & $\begin{array}{c}6 \text { weeks } \\
\text { after treatment }\end{array}$ & $\begin{array}{l}\text { Before } \\
\text { treatment }\end{array}$ & $\begin{array}{c}6 \text { weeks } \\
\text { after treatment }\end{array}$ & $\begin{array}{c}\text { Before } \\
\text { treatment }\end{array}$ & $\begin{array}{c}6 \text { weeks } \\
\text { after treatment }\end{array}$ \\
\hline Normal control & 8 & $5.3 \pm 1.12$ & $5.2 \pm 1.53$ & $19.85 \pm 3.21$ & $18.11 \pm 3.22$ & $7.1 \pm 1.21$ & $6.9 \pm 1.42$ \\
\hline Vehicle & 7 & $16.8 \pm 1.21^{\mathrm{a}}$ & $17.3 \pm 1.12$ & $61.21 \pm 4.12^{\mathrm{a}}$ & $75.91 \pm 5.12$ & $10.3 \pm 2.11^{\mathrm{a}}$ & $13.23 \pm 2.34$ \\
\hline DBT-L & 8 & $17.3 \pm 0.98^{\mathrm{a}}$ & $12.2 \pm 1.31^{\mathrm{b}, \mathrm{c}}$ & $62.31 \pm 3.52^{\mathrm{a}}$ & $52.11 \pm 2.34^{\mathrm{b}, \mathrm{c}}$ & $11.8 \pm 2.13^{\mathrm{a}}$ & $9.21 \pm 1.02^{\mathrm{b}, \mathrm{c}}$ \\
\hline DBT-M & 9 & $17.1 \pm 1.11^{\mathrm{a}}$ & $13.1 \pm 1.51^{\mathrm{b}, \mathrm{c}}$ & $63.11 \pm 2.32^{\mathrm{a}}$ & $49.13 \pm 1.28^{b, c}$ & $12.4 \pm 2.29^{\mathrm{a}}$ & $9.11 \pm 1.13^{\mathrm{b}, \mathrm{c}}$ \\
\hline DBT-H & 9 & $16.9 \pm 1.02^{\mathrm{a}}$ & $13.4 \pm 1.22^{\mathrm{b}, \mathrm{c}}$ & $64.10 \pm 3.13^{\mathrm{a}}$ & $46.32 \pm 4.19^{b, c}$ & $13.9 \pm 2.01^{\mathrm{a}}$ & $8.32 \pm 0.12^{b, c}$ \\
\hline Gliclazide & 9 & $17.2 \pm 0.92^{\mathrm{a}}$ & $10.3 \pm 0.42^{\mathrm{b}, \mathrm{c}}$ & $63.89 \pm 4.11^{\mathrm{a}}$ & $43.12 \pm 4.43^{\mathrm{b}, \mathrm{c}}$ & $13.4 \pm 2.32^{\mathrm{a}}$ & $7.12 \pm 2.11^{\mathrm{b}, \mathrm{c}}$ \\
\hline
\end{tabular}

Three rats in the vehicle group and one in the DBT-L group died during the experiment and were not included in the analysis. Data are expressed as the mean \pm standard deviation. Student's t-test and one-way analysis of variance were used to analyze the differences between the two groups and among the six groups, respectively. ${ }^{a} \mathrm{P}<0.05 \mathrm{vs}$. normal control group; ${ }^{\mathrm{b}} \mathrm{P}<0.05$ vs. vehicle group; ${ }^{\mathrm{c}} \mathrm{P}<0.05 \mathrm{vs}$. before treatment. DBT, Danggui Buxue Tang; DBT-L, low concentration (0.5 kg/l) DBT; DBT-M, medium concentration (1.0 kg/l) DBT; DBT-H, high concentration $(2.0 \mathrm{~kg} / \mathrm{l}) \mathrm{DBT}$.

Table II. Effects of DBT on serum and urine $\beta 2$-microglobulin levels and type IV collagen expression in rats.

\begin{tabular}{|c|c|c|c|c|c|c|c|}
\hline \multirow[b]{2}{*}{ Group } & \multirow[b]{2}{*}{$\mathrm{n}$} & \multicolumn{2}{|c|}{$\begin{array}{c}\text { Serum } \\
\beta 2-\text { microglobulin }(\mathrm{mg} / \mathrm{l})\end{array}$} & \multicolumn{2}{|c|}{$\begin{array}{c}\text { Urine } \\
\beta 2 \text {-microglobulin }(\mu \mathrm{g} / \mathrm{l})\end{array}$} & \multicolumn{2}{|c|}{$\begin{array}{c}\text { Type IV } \\
\text { collagen }(\mathrm{ng} / \mathrm{ml})\end{array}$} \\
\hline & & $\begin{array}{l}\text { Before } \\
\text { treatment }\end{array}$ & $\begin{array}{c}6 \text { weeks } \\
\text { after treatment }\end{array}$ & $\begin{array}{l}\text { Before } \\
\text { treatment }\end{array}$ & $\begin{array}{c}6 \text { weeks } \\
\text { after treatment }\end{array}$ & $\begin{array}{c}\text { Before } \\
\text { treatment }\end{array}$ & $\begin{array}{c}6 \text { weeks } \\
\text { after treatment }\end{array}$ \\
\hline Normal control & 8 & $2.01 \pm 0.12$ & $2.41 \pm 0.32$ & $7.41 \pm 0.18$ & $7.01 \pm 0.32$ & $21.1 \pm 2.42$ & $23.2 \pm 3.51$ \\
\hline Vehicle & 7 & $7.01 \pm 0.22^{\mathrm{a}}$ & $8.08 \pm 0.13$ & $14.31 \pm 0.22^{\mathrm{a}}$ & $13.06 \pm 0.41$ & $37.3 \pm 2.23^{\mathrm{a}}$ & $40.1 \pm 3.41$ \\
\hline DBT-L & 8 & $6.95 \pm 0.41^{\mathrm{a}}$ & $5.41 \pm 0.13^{b}$ & $13.64 \pm 0.32^{\mathrm{a}, \mathrm{c}}$ & $10.01 \pm 0.12^{b, c}$ & $36.3 \pm 4.41^{\mathrm{a}}$ & $27.1 \pm 4.01^{\mathrm{b}, \mathrm{c}}$ \\
\hline DBT-M & 9 & $6.78 \pm 0.33^{\mathrm{a}}$ & $5.01 \pm 0.44^{\mathrm{b}}$ & $13.01 \pm 0.37^{\mathrm{a}, \mathrm{c}}$ & $9.94 \pm 0.32^{\mathrm{b}, \mathrm{c}}$ & $34.7 \pm 3.22^{\mathrm{a}}$ & $28.1 \pm 2.90^{\mathrm{b}, \mathrm{c}}$ \\
\hline DBT-H & 9 & $7.01 \pm 0.35^{\mathrm{a}}$ & $5.13 \pm 0.61^{b}$ & $12.75 \pm 0.28^{\mathrm{a}, \mathrm{c}}$ & $9.01 \pm 0.14^{\mathrm{b}, \mathrm{c}}$ & $35.1 \pm 2.12^{\mathrm{a}}$ & $25.1 \pm 3.21^{\mathrm{b}, \mathrm{c}}$ \\
\hline Gliclazide & 9 & $7.03 \pm 0.22^{\mathrm{a}}$ & $6.78 \pm 0.12^{b}$ & $13.01 \pm 0.42^{\mathrm{a}, \mathrm{c}}$ & $10.01 \pm 0.32^{b, c}$ & $33.9 \pm 3.41^{\mathrm{a}}$ & $27.1 \pm 2.42^{\mathrm{b}, \mathrm{c}}$ \\
\hline
\end{tabular}

Three rats in the vehicle group and one in the DBT-L group died during the experiment and were not included in the analysis. Data are expressed as the mean \pm standard deviation. Student's t-test and one-way analysis of variance were used to analyze the differences between the two groups and among the six groups, respectively ${ }^{a} \mathrm{P}<0.05$ vs. normal control group; ${ }^{b} \mathrm{P}<0.05$ vs. vehicle group; ${ }^{\mathrm{c}} \mathrm{P}<0.05$ vs. before treatment. DBT, Danggui Buxue Tang; DBT-L, low concentration (0.5 kg/l) DBT; DBT-M, medium concentration (1.0 kg/l) DBT; DBT-H, high concentration $(2.0 \mathrm{~kg} / \mathrm{l}) \mathrm{DBT}$.

Wuhan Boster Biological Technology, Ltd.) at $4^{\circ} \mathrm{C}$ overnight. The sections were incubated with biotinylated goat anti-rabbit IgG antibody as the secondary antibody (1:100; BA1003; Wuhan Boster Biological Technology, Ltd.). A slide known to be positive to HPA (Wuhan Biological Engineering Co., Ltd.) was set as a positive control, and an irrelevant isotype rabbit IG was used for a negative control. The peroxidase reaction was visualized using 3,3'-diaminobenzidine tetrahydrochloride (Wuhan Boster Biological Technology, Ltd.) as a peroxidase substrate.

Images of immunostained sections were captured using a Canon 1000D digital camera (Canon, Inc., Tokyo, Japan). The positive immunostaining, which presented as brown or yellow granules in the cytoplasm and/or cell membrane, was automatically detected and measured for the staining intensity and area using a computer equipped with HPIAS-1000 image analysis software (Wuhan Qingping Image Technology, Co., Ltd., Wuhan, China). Five visual fields were randomly selected and assessed for immunoreactive areas at x200 magnification. The ratio (\%) of the HPA-positive glomerular area to the total glomerular area assessed was calculated, and the mean ratio of the five fields was defined to represent the level of HPA expression.

Statistical analysis. All statistical analyses were performed using SPSS software, version 13.0 (SPSS, Inc., Chicago, IL, USA). Quantitative data are presented as the mean \pm standard deviation. Student's t-test and one-way analysis of variance methods were used for analyzing the differences between two groups and among the six groups, respectively. $\mathrm{P}<0.05$ was considered to indicate a statistically significant difference. 


\section{Results}

General condition of the animals. Following the injection of STZ, six rats died or failed to develop diabetes. Thus, the 46 rats that developed STZ-induced diabetes mellitus were randomly distributed into the Vehicle $(n=10)$, DBT-H $(n=9)$, DBT-M (n=9), DBT-L $(n=9)$ and Gliclazide $(n=9)$ groups. The numbers of rats that died during the next six weeks were three in Vehicle group and one in the DBT-L group. After six weeks of high-glucose diet, the diabetic rats in the Vehicle group showed typical diabetic symptoms, including polydipsia, polyphagia, polyuria and body weight loss, with dull fur and reduced activity.

Effects of DBT on FBG, BUN, Scr, serum and urine $\beta 2-M G$, and type IV collagen expression. All rats with STZ-induced diabetes mellitus had elevated levels of FBG, BUN, Scr, serum and urine $\beta 2-\mathrm{MG}$, and type IV collagen expression prior to treatment compared with rats in the $\mathrm{NC}$ group (all $\mathrm{P}<0.05$; Tables I and II). Treatment with gliclazide for six weeks significantly decreased the levels of FBG, BUN, Scr, serum and urine $\beta 2-\mathrm{MG}$, and type IV collagen expression, as compared with the vehicle group $(\mathrm{P}<0.05)$. Notably, DBT treatment at different doses resulted in a similar reversal of these abnormal parameters, although they were all above the normal levels.

DBT treatment restores the changes in the glomerular ultrastructure in diabetic rats. To assess the protective effects on the renal glomerulus, ultrastructural analysis by electron microscopy was performed in the renal cortex of diabetic rats after six weeks of DBT treatment, with the untreated diabetic and normal rats as controls. All rats in NC group showed normal glomerular ultrastructure. Specifically, numerous thick primary processes extended from the cell body of the podocytes. These then further ramified into fine, finger-like secondary foot processes, which interdigitated with foot processes from adjacent podocytes. The foot processes formed a fence-like structure which was closely attached to the GBM. Together with the GBM, the endothelium and podocytes formed the filtration barrier, and the mesangium consisting of GMCs and the matrix lied between the glomerular capillaries (Fig. 1A). In the Vehicle group, the secondary foot processes of podocytes became irregular and displayed numerous areas of effacement and GBM thickened with evident expansion of GMCs (Fig. 1B). However, in rats after six weeks of DBT treatment, there was a notable improvement in the ultrastructural abnormalities (Fig. 1C-E), particularly in those in the DBT-H group, where GBM thickness, podocytes and GMCs were similar to those in the NC group. Similar improvement in the ultrastructural abnormalities was observed in rats in the Gliclazide group (Fig. 1F).

DBT treatment decreases HPA expression in the renal cortex in diabetic rats. The positive immunohistochemical staining of HPA protein was shown as brown signals in the cytoplasm and/or cell membrane of podocytes, mesangial cells and tubular epithelial cells. The positive signals were quantified using HPIAS-1000 image analysis software, and the average values of HPA immunohistochemical staining in the different groups are shown in Table III. HPA expression was negative
Table III. Expression of HPA in the kidney tissues.

\begin{tabular}{lcc}
\hline Group & $\mathrm{n}$ & HPA expression $(\%)$ \\
\hline Normal control & 8 & $18.00 \pm 8.2$ \\
Vehicle & 7 & $109.50 \pm 18.95^{\mathrm{a}}$ \\
DBT-L & 8 & $71.00 \pm 8.04^{\mathrm{a}-\mathrm{c}}$ \\
DBT-M & 9 & $55.50 \pm 1.29^{\mathrm{a}, \mathrm{b}}$ \\
DBT-H & 9 & $36.25 \pm 3.86^{\mathrm{a}-\mathrm{c}}$ \\
Gliclazide & 9 & $52.00 \pm 2.71^{\mathrm{a}, \mathrm{b}}$
\end{tabular}

Three rats in the Vehicle group and one in the DBT-L group died during the experiment and were not included in the analysis. Data are expressed as the mean \pm standard deviation. Student's t-test and one-way analysis of variance were used to analyze the differences between the two groups and among the six groups, respectively ${ }^{\mathrm{a} P}<0.05$ vs. normal control group; ${ }^{\mathrm{b}} \mathrm{P}<0.05$ vs. Vehicle group; ${ }^{\mathrm{c}} \mathrm{P}<0.05$ vs. Gliclazide group. DBT, Danggui Buxue Tang; DBT-L, low concentration $(0.5 \mathrm{~kg} / \mathrm{l})$ DBT; DBT-M, medium concentration $(1.0 \mathrm{~kg} / \mathrm{l})$ DBT; DBT-H, high concentration $(2.0 \mathrm{~kg} / \mathrm{l}) \mathrm{DBT}$.

or very low in glomeruli in the control group (Table III and Fig. 2A). The rats in the Vehicle group exhibited a significantly higher HPA immunostaining in the glomeruli (Table III and Fig. 2B). However, HPA protein expression was significantly and dose-dependently decreased in the DBT groups, as compared to the Vehicle group (Table III and Fig. 2C-E). HPA protein expression was also significantly decreased in the Gliclazide group, as compared to the Vehicle group (Table III and Fig. 2F). Notably, the high-dose DBT $(2.0 \mathrm{~kg} / \mathrm{l})$ treatment showed an increased effect compared with gliclazide with respect to decreasing HPA expression (36.25 \pm 3.86 vs. $52.00 \pm 2.71 ; \mathrm{P}<0.05)$.

\section{Discussion}

Development of diabetic nephropathy is characterized by increased plasma levels of creatinine and BUN in STZ-induced diabetic rats (22). STZ is a powerful alkylating agent that has been widely accepted as an inducer to produce diabetes mellitus in rodents (23). STZ interferes with numerous cellular processes, such as glucose transport and glucokinase function, and may induce DNA strand breakage (24). A single large dose of STZ (such as $30 \mathrm{mg} / \mathrm{kg}$ ) may induce diabetes mellitus in rodents; however, this dose has been associated with high mortality. In the present study, a two-week high-glucose diet followed by a single injection of $30 \mathrm{mg} / \mathrm{kg}$ STZ induced diabetes mellitus in $88 \%$ (46/52) of the surviving rats. Moreover, the subsequent six-week maintenance of the high-glucose diet resulted in typical features of nephropathy in the diabetic rats. Besides symptoms of hyperglycemia and obesity, this model appeared to exhibit the most consistent and robust increases in BUN and Scr; as such, it has been used as a useful model of progressive diabetic renal disease.

The pathological characteristics of diabetic nephropathy include the proliferation of GMCs, thickening of the GBM, and accumulation of ECM proteins, followed by progressive glomerulosclerosis and worsening of renal function (25-27). An in vitro study has shown that GMCs are a major source 

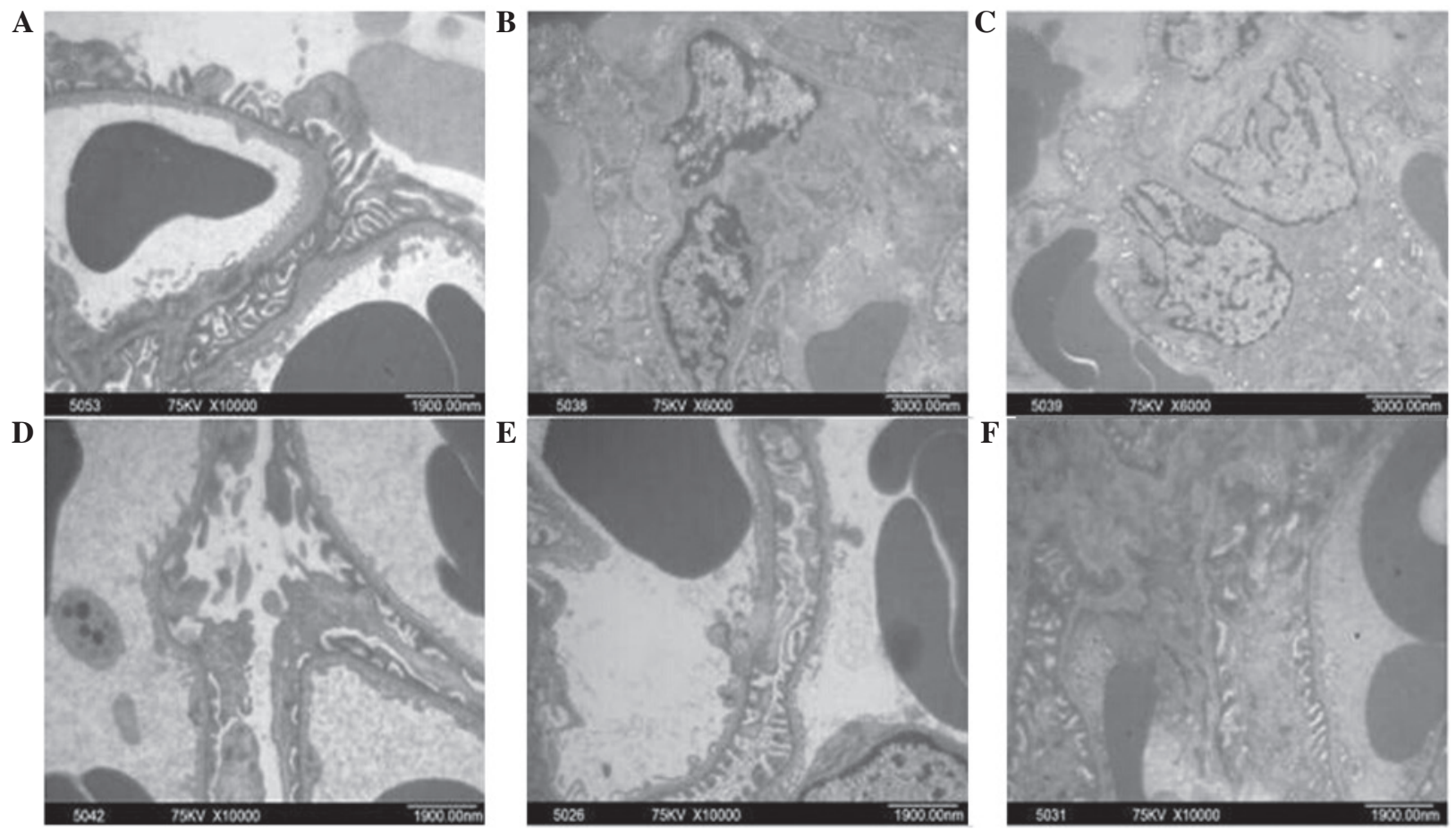

Figure 1. Restoration of the glomerular ultrastructural changes by Danggui Buxue Tang (DBT) treatment in rats with streptozotocin-induced diabetes mellitus. Representatives of the glomerular ultrastructure as detected using electron microscopy in (A) untreated normal control, (B) vehicle-treated, (C) $0.5 \mathrm{~kg} / \mathrm{l}$ DBT-treated, (D) $1.0 \mathrm{~kg} / 1$ DBT-treated, (E) $2.0 \mathrm{~kg} / 1$ DBT-treated and (F) gliclazide-treated rats. The renal injury was substantially improved after six weeks of DBT treatment in the DBT-L, DBT-M and DBT-H groups.
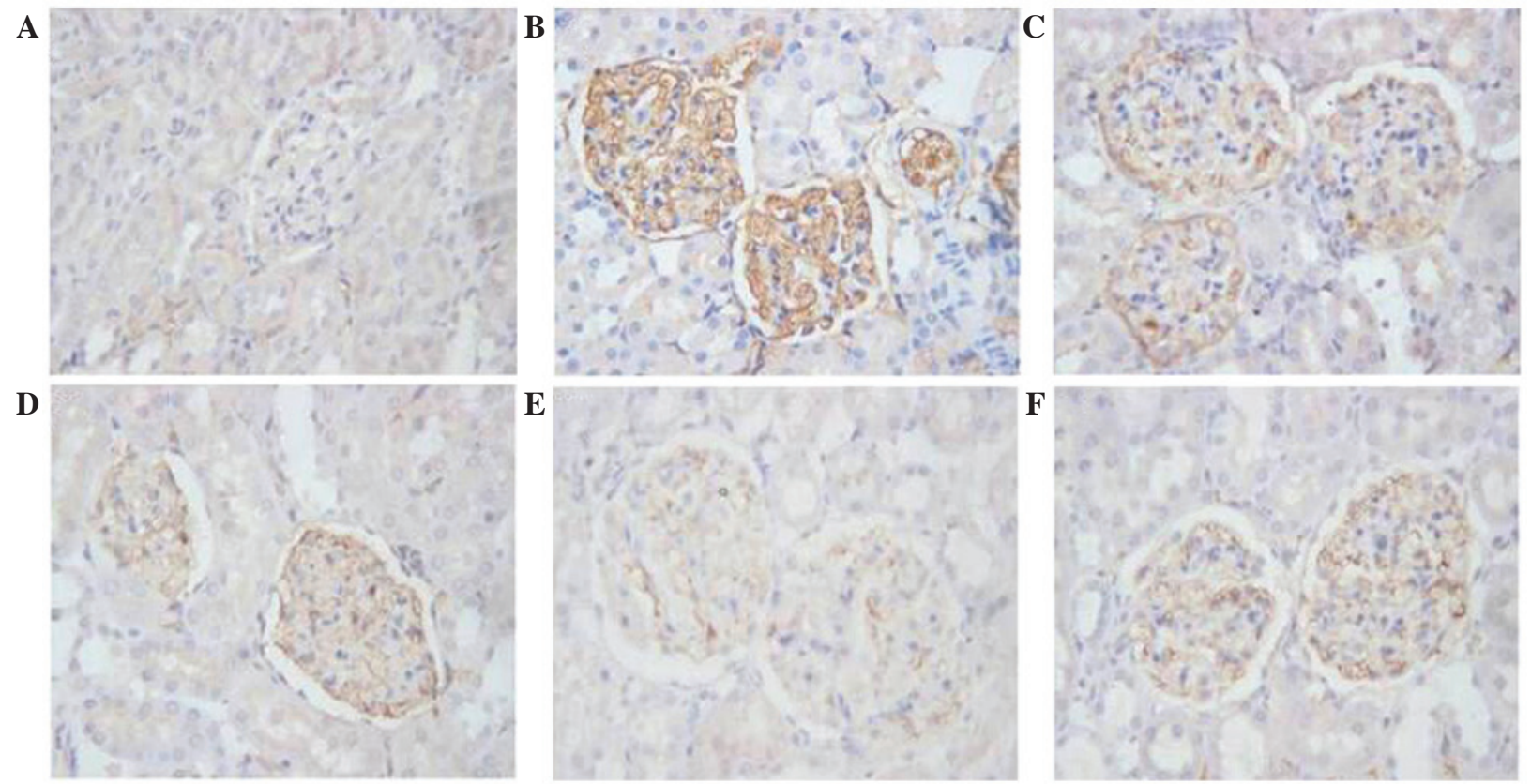

Figure 2. Immunohistochemical analysis for heparanase expression in the kidney tissues of the (A) untreated normal control, (B) vehicle-treated, (C) $0.5 \mathrm{~kg} / \mathrm{l}$ Danggui Buxue Tang (DBT)-treated, (D) $1.0 \mathrm{~kg} / 1$ DBT-treated, (E) $2.0 \mathrm{~kg} / 1$ DBT-treated and (F) gliclazide-treated rats (magnification, x200).

of free radicals following exposure to high glucose concentrations (28). Hyperglycemia causes oxidative stress and leads to an increase in a number of complements, growth factors, and cytokines, such as transforming growth factor (TGF)- $\beta$, and the excessive production and accumulation of ECM proteins (29). This increased production in GMCs has been implicated in the development of diabetic nephropathy. Our previous in vitro study showed that high glucose induced an increased synthesis of various ECM proteins, including type IV collagen, laminin, and fibronectin, in glomerular mesangial and epithelial cells (13). Furthermore, in the present study, the high glucose-induced diabetic rats displayed abnormal thickening of the GBM, loss of podocytes, effacement of foot processes and expansion of mesangial cells. These findings 
clearly indicate that the high glucose not only induces glomerular mesangial and epithelial proliferation, but also causes ultrastructural changes of the kidney.

Radix Astragali (Huangqi) is widely used in China, and has been studied in animal models and patients with various renal diseases for its effects on cytokines and reactive oxygen species, ischemia/reperfusion injury and mechanisms of renal fibrosis (30). A systematic review revealed that Radix Astragali and its effective components are may be able to FBG and albuminuria levels, reversing the glomerular hyperfiltration and ameliorating the pathological changes associated with early diabetic nephropathy in rat models (31). DBT is traditionally used to treat gynecological disorders and hypertension in China. DBT alone appears to improve the renal functions and increase renal BMP-7 expression in STZ-induced diabetic rats (27). The present results showed that DBT was effective at improving diabetic nephropathy. Similar to the action of gliclazide, DBT treatment effectively attenuated the increases in FBG, BUN, Scr, and serum and urine $\beta 2-\mathrm{MG}$ in rats with STZ-induced diabetes mellitus. The accumulation of ECM proteins, including type IV collagen, plays an important role in the pathogenesis of diabetic nephropathy (13). In the present study, DBT treatment caused a reduction in type IV collagen expression compared with the Vehicle group, suggesting that DBT may prevent kidney damage by reducing the increase of ECM proteins.

DBT in combination with Radix Astragali accelerates histological recovery of the kidney, and delays the progression of renal fibrosis as much as the acetyl choline inhibitor enalapril (32). This appeared to occur via the suppression of TGF- $\beta$ expression and mitigation of the upregulated expression of type III and IV collagens, fibronectin and laminin in a rat model of chronic puromycin-induced nephrosis (33). In the present study, DBT treatment substantially improved diabetes-induced structural cellular alterations, such as the proliferation of GMCs, loss of podocyte foot processes and abnormal thickness of the GBM. In particular, the GBM thickness, numbers of podocytes and GMCs were similar between the DBT-H and NC groups. These results further support those obtained in our previous in vitro study in which DBT had inhibitory effects on high glucose-induced GMC proliferation and the expression of ECM proteins (13).

Heparan sulfate proteoglycans (HSPG) are ubiquitous macromolecules associated with cell surfaces and ECM, and represent a major constituent of the GBM (34). HSPG in the kidneys are composed of an agrin protein core that is covalently linked to the negatively charged glycosaminoglycan heparan sulfate (35). HPA is an endoglycosidase which cleaves heparan sulfate and hence participates in the degradation and remodeling of the ECM. A previous study indicated that increased glomerular expression of HPA was associated with overt diabetic nephropathy (36). In addition, the expression of HPA was upregulated in animal models of proteinuric renal disease, including passive heymann nephritis, puromycin nephrosis, anti-GBM nephritis and adriamycin nephropathy (37). Furthermore, the overexpression of HPA in transgenic mice led to proteinuria, while treatment with a polyclonal anti-HPA antibody resulted in a three-fold reduction of proteinuria in a model of anti-GBM disease (37). In the present study, the expression of HPA in the diabetic rat renal cortex was evaluated using immunohistochemistry to investigate the effect of DBT treatments on HPA expression. HPA expression was negative or low in the glomeruli of the NC group. However, the rats in the Vehicle group exhibited a significantly higher HPA immunostaining in the glomeruli. These findings were consistent with our previous in vitro study, which demonstrated that high glucose was able to induce HPA expression in podocytes and a reduction of cell surface heparan sulfate (38). Overexpression of HPA may lead to an increased permeability of the GBM to macromolecules, due to the degradation of glomerular heparan sulfate and the activation of a variety of complements and cytokines (39). To the best of our knowledge, the present study is the first to demonstrate that DBT treatment effectively inhibited the levels of HPA expression in renal glomerular mesangium, indicating that DBT may preserve renal functional and structural integrity. This may at least partially occur via the inhibition of HPA expression, resulting in increased heparin sulfate expression in the renal glomerular mesangium. Notably, the high-dose DBT $(2 \mathrm{~kg} / \mathrm{l})$ treatment was more effective than gliclazide with respect to decreasing HPA expression. However, heparan sulfate loss in proteinuric renal disease may be attributed to a number of mechanisms other than HPA, including depolymerization of heparin sulfate by increasing the formation of free radicals, such as reactive oxygen species (ROS) and decreasing antioxidant capacity (40). Oxidative stress may facilitate the formation of advanced glycation end products, which is a pathogenic factor in sustained hyperglycemia-induced kidney injuries (41). DBT has been reported to be a native free radical scavenger, and is able to decrease hyperglycemia-induced ROS generation in the kidneys of diabetic rats or in mesangial cells (28). Therefore, anti-ROS treatments such as DBT may prevent hyperglycemia-induced renal damage in diabetes mellitus.

It should be noted that the present report is only a preliminary observational study. Thus, further studies are required to identify the specific active components present in DBT that are responsible for the beneficial effects on the renal function, and to elucidate the underlying mechanisms by which DBT improves and prevents diabetes mellitus and diabetic nephropathy.

DBT appears to be a renal protective agent by reversing renal ultrastructural changes in a high-glucose/STZ-induced diabetic nephropathy rat model. Furthermore, DBT exhibits beneficial effects by decreasing the elevated HPA expression in the renal glomerular mesangium. Therefore, DBT is potentially a novel therapeutic intervention for the treatment of diabetic nephropathy.

\section{Acknowledgements}

This study was supported by funding from the National Natural Science Foundation of China (grant no. 30973819).

\section{References}

1. Heerspink HJ and de Zeeuw D: The kidney in type 2 diabetes therapy. Rev Diabet Stud 8: 392-402, 2011.

2. Abuissa $\mathrm{H}$ and O'Keefe J Jr: The role of renin-angiotensin-aldosterone system-based therapy in diabetes prevention and cardiovascular and renal protection. Diabetes Obes Metab 10: 1157-1166, 2008.

3. Zhang WL, Zheng KY, Zhu KY, Zhan JY, Bi CW, Chen JP, Dong TT, Choi RC, Lau DT and Tsim KW: Chemical and biological assessment of angelica roots from different cultivated regions in a chinese herbal decoction danggui buxue tang. Evid Based Complement Alternat Med 2013: 483286, 2013. 
4. Wu YC and Hsieh CL: Pharmacological effects of Radix Angelica sinensis (Danggui) on cerebral infarction. Chin Med 6: 32, 2011.

5. Wang Z, Wang $\mathrm{J}$ and Chan P: Treating type 2 diabetes mellitus with traditional chinese and Indian medicinal herbs. Evid Based Complement Alternat Med 2013: 343594, 2013.

6. Toda S, Yase Y and Shirataki Y: Inhibitory effects of astragali radix, crude drug in Oriental medicines on lipid peroxidation and protein oxidative modification of mouse brain homogenate by copper. Phytother Res 14: 294-296, 2000.

7. Gao QT, Choi RC, Cheung AW, Zhu JT, Li J, Chu GK, Duan R, Cheung JK, Jiang ZY, Dong XB, et al: Danggui buxue tang - a Chinese herbal decoction activates the phosphorylations of extracellular signal-regulated kinase and estrogen receptor alpha in cultured MCF-7 cells. FEBS Lett 581: 233-240, 2007.

8. Zhang YW, Xie D, Xia B, Zhen RT, Liu IM and Cheng JT: Suppression of transforming growth factor-betal gene expression by Danggui buxue tang, a traditional Chinese herbal preparation, in retarding the progress of renal damage in streptozotocin-induced diabetic rats. Horm Metab Res 38: 82-88, 2006

9. Zhang YW, Xie D, Chen YX, Zhang HY and Xia ZX: Protective effect of Gui Qi mixture on the progression of diabetic nephropathy in rats. Exp Clin Endocrinol Diabetes 114: 563-568, 2006.

10. Ha TS: Roles of adaptor proteins in podocyte biology. World J Nephrol 2: 1-10, 2013.

11. Chiang YY, Takebayashi S and Oberley TD: In vitro analysis of extracellular matrix production by porcine glomerular mesangial and vascular smooth muscle cells. Am J Pathol 138: 1349-1358, 1991.

12. Lan T, Liu W, Xie X, Xu S, Huang K, Peng J, Shen X, Liu P, Wang L, Xia P and Huang H: Sphingosine kinase-1 pathway mediates high glucose-induced fibronectin expression in glomerular mesangial cells. Mol Endocrinol 25: 2094-2105, 2011

13. Ke HL, Zhang YW, Zhou BF and Zhen RT: Effects of Danggu Buxue Tang, a traditional Chinese herbal decoction, on high glucose-induced proliferation and expression of extracellular matrix proteins in glomerular mesangial cells. Nat Prod Res 26 1022-1026, 2012

14. Bame KJ: Heparanases: endoglycosidases that degrade heparan sulfate proteoglycans. Glycobiology 11: 91R-98R, 2011

15. Jiang P, Kumar A, Parrillo JE, Dempsey LA, Platt JL, Prinz RA and $\mathrm{Xu} X$ : Cloning and characterization of the human heparanase-1 (HPR1) gene promoter: Role of GA-binding protein and Sp1 in regulating HPR1 basal promoter activity. J Biol Chem 277: 8989-8998, 2002.

16. Gil N, Goldberg R, Neuman T, Garsen M, Zcharia E, Rubinstein AM, van Kuppevelt T, Meirovitz A, Pisano C, Li JP, et al: Heparanase is essential for the development of diabetic nephropathy in mice. Diabetes 61: 208-216, 2012

17. Zhang YW, Wu CY and Cheng JT: Merit of Astragalus polysaccharide in the improvement of early diabetic nephropathy with an effect on mRNA expressions of NF-kappaB and IkappaB in renal cortex of streptozotoxin-induced diabetic rats. J Ethnopharmacol 114: 387-392, 2007.

18. Clark JD, Gebhart GF, Gonder JC, Keeling ME and Kohn DF: Special report: Guide for the care and use of laboratory animals, ILAR J 1997: 41-48, 1997.

19. Nakamura T, Terajima T, Ogata T, Ueno K, Hashimoto N, Ono K and Yano S: Establishment and pathophysiological characterization of type 2 diabetic mouse model produced by streptozotocin and nicotinamide. Biol Pharm Bull 29: 1167-1174, 2006.

20. Schernthaner G, Grimaldi A, Di Mario U, Drzewoski J, Kempler P, Kvapil M, Novials A, Rottiers R, Rutten GE and Shaw KM: GUIDE study: Double-blind comparison of once-daily gliclazide MR andglimepiride in type 2 diabetic patients. Eur J Clin Invest 34: 535-542, 2004.

21. U.S. Food and Drug Administration: Guidance for Industry and Reviewers: Estimating the Safe Starting Dose in Clinical Trials for Therapeutics in Adult Healthy Volunteers. December 2002
22. Wang GG, Lu XH, Li W, Zhao X and Zhang C: Protective effects of luteolin on diabetic nephropathy in STZ-induced diabetic rats. Evid Based Complement Alternat Med 2011: 323171, 2011.

23. King AJ: The use of animal models in diabetes research. Br J Pharmacol 166: 877-894, 2012

24. Like AA and Rossini AA: Streptozotocin-induced pancreatic insulitis: New model of diabetes mellitus. Science 193: 415-417, 1976.

25. Kanwar YS, Wada J, Sun L, Xie P, Wallner EI, Chen S, Chugh S and Danesh FR: Diabetic nephropathy: Mechanisms of renal disease progression. Exp Biol Med (Maywood) 233: 4-11, 2008.

26. Wolf G: New insights into the pathophysiology of diabetic nephropathy: From haemodynamics to molecular pathology. Eur J Clin Invest 34: 785-796, 2004.

27. Dronavalli S, Duka I and Bakris GL: The pathogenesis of diabetic nephropathy. Nat Clin Pract Endocrinol Metab 4: 444-452, 2008.

28. Yeh CH, Chang CK, Cheng KC, Li YX, Zhang YW and Cheng JT: Role of bone morphogenetic proteins-7 (BMP-7) in the renal improvement effect of DangGui (Angelica sinensis) in type-1 diabetic rats. Evid Based Complement Alternat Med 2011: 796723, 2011

29. Giacco F and Brownlee M: Oxidative stress and diabetic complications. Circ Res 107: 1058-1070, 2010.

30. Zhong Y, Deng Y, Chen Y, Chuang PY and Cijiang He J: Therapeutic use of traditional Chinese herbal medications for chronic kidney diseases. Kidney Int 84: 1108-1118, 2013.

31. ZhangJ,XieX,LiCandFuP: Systematic review of therenalprotective effect of Astragalus membranaceus (root) on diabetic nephropathy in animal models. J Ethnopharmacol 126: 189-196, 2009.

32. Chen X, Cao A, Wang L, Yin P, Zhang X and Peng W: Prevention and treatment of diabetic nephropathy using traditional Chinese medicine. J Integr Nephrol Androl 1: 53-57, 2014.

33. Wang H, Li J, Yu L, Zhao Y and Ding W: Antifibrotic effect of the Chinese herbs, Astragalus mongholicus and Angelica sinensis, in a rat model of chronic puromycin aminonucleoside nephrosis. Life Sci 74: 1645-1658, 2004

34. Shimomura H and Spiro RG: Studies on macromolecular components of human glomerular basement membrane and alterations in diabetes. Decreased levels of heparan sulfate proteoglycan and laminin. Diabetes 36: 374-381, 1987.

35. Rops AL, van der Vlag J, Lensen JF, Wijnhoven TJ, van den Heuvel LP, van Kuppevelt TH, Berden JH: Heparan sulfate proteoglycans in glomerular inflammation.Kidney Int 65: 768-785, 2004

36. van den Hoven MJ, Rops AL, Bakker MA, Aten J, Rutjes N, Roestenberg P, Goldschmeding R, Zcharia E, Vlodavsky I, van der Vlag J and Berden JH: Increased expression of heparanase in overt diabetic nephropathy. Kidney Int 70: 2100-2108, 2006.

37. Shafat I, Agbaria A, Boaz M, Schwartz D, Baruch R, Nakash R, Ilan N, Vlodavsky I and Weinstein T: Elevated urine heparanase levels are associated with proteinuria and decreased renal allograft function. PLoS One 7: e44076, 2012.

38. Maxhimer JB, Somenek M, Rao G, Pesce CE, Baldwin D Jr, Gattuso P, Schwartz MM, Lewis EJ, Prinz RA and Xu X: Heparanase-1 gene expression and regulation by high glucose in renal epithelial cells: A potential role in the pathogenesis of proteinuria in diabetic patients. Diabetes 54: 2172-2178, 2005.

39. van den Hoven MJ, Rops AL, Bakker MA, Aten J, Rutjes N, Roestenberg P, Goldschmeding R, Zcharia E, Vlodavsky I, van der Vlag J and Berden JH: Increased expression of heparanase in overt diabetic nephropathy. Kidney Int 70: 2100-2108, 2006.

40. Kramer A, van den Hoven M, Rops A, Wijnhoven T, van den Heuvel L, Lensen J, van Kuppevelt T, van Goor H, van der Vlag J, Navis G and Berden JH: Induction of glomerular heparanase expression in rats with adriamycin nephropathy is regulated by reactive oxygen species and the renin-angiotensin system. J Am Soc Nephrol 17: 2513-2520, 2006.

41. Tan AL, Forbes JM and Cooper ME: AGE, RAGE and ROS in diabetic nephropathy. Semin Nephrol 27: 130-143, 2007. 